\title{
Application of Humanized Nursing in Uterine Artery Embolization for Patients of Uterine Fibroids
}

\author{
Huijuan Cai, Lirong Mo, Xiaojuan Liao* \\ Gynecology Department, The First Affiliated Hospital of Jinan University, Guangzhou, China \\ Email address: \\ 15975482120@163.com (Huijuan Cai),L8808104@126.com (Xiaojuan Liao),804893466@qq.com (Lirong Mo) \\ ${ }^{*}$ Corresponding author
}

To cite this article:

Huijuan Cai, Lirong Mo, Xiaojuan Liao. Application of Humanized Nursing in Uterine Artery Embolization for Patients of Uterine Fibroids. American Journal of Nursing Science. Vol. 10, No. 1, 2021, pp. 55-58. doi: 10.11648/j.ajns.20211001.20

Received: January 23, 2021; Accepted: January 30, 2021; Published: February 9, 2021

\begin{abstract}
Background: Uterine artery embolization (UAE) has been widely used to treat uterine fibroids in recent years, which is less invasive and preserving the patient's uterus. Anyway, the patient could still have negative emotions in the perioperative period because of pain, vaginal bleeding, psychological stress, or other factors. High-quality humanized nursing is needed to improve patients' situations. Object: To analyze the application and effect of humanized nursing in UAE for patients of uterine fibroids. Method: 90 patients with uterine fibroids admitted to our hospital from November 2019 to November 2020 were selected and divided into two groups according to a random drawing. The patients in the control group were given traditional nursing care. The patients in the observation group were given humanized nursing mode on this basis. The anxiety and depression degree before being discharged from hospital, satisfaction with different nursing modes, QOL score, amenorrhea, anemia, infection, arterial thrombosis, and other adverse conditions of the two groups were investigated. Result: The QOLS of the observation group after nursing were significantly higher than those of the control group, and the differences were statistically significant $(P<0.05)$. The score of HAMA and HAMD of the observation group was significantly higher than that of the control group, and the differences were statistically significant $(P<0.05)$. The satisfaction of the observation group was significantly higher than that of the control group, and the differences were statistically significant $(P<0.05)$. The rate of adverse complications of the observation group was significantly lower than that of the control group, and the differences were statistically significant $(P<0.05)$. Conclusion: The application of a humanized nursing model for the care of patients with UAE for uterine fibroids can effectively alleviate and eliminate negative perioperative emotions and significantly improve depression, which in turn is conducive to improving their postoperative quality of life, with high patient satisfaction and fewer cases of adverse reactions, with a high degree of safety, and is worth promoting in the clinic.
\end{abstract}

Keywords: Humanized Care, Uterine Fibroids, Uterine Leiomyoma, Uterine Artery Embolization, Quality of Life Score

\section{Introduction}

Uterine fibroids are kinds of common gynecological disease, which is a benign tsumor that primarily affects women of childbearing age. The main symptoms of uterine fibroids are excessive menstruation, prolonged menstruation, pelvic pain, and compression pain of neighboring organs, which could affect the quality of daily life and work if not treated promptly [1]. At present, clinical treatment methods include myomectomy, drug therapy, and other methods [2]. Among them, uterine artery embolization (UAE) has the advantages of less trauma, quick recovery, simple operation, and high uterine retention rate, which is recognized by most patients and has been more and more widely clinical applied $[3,4]$. Humanized nursing is a modern patient-centered nursing model. It is an effective, holistic, individual, and creative nursing model that gradually replaces the traditional disease-centered nursing model in clinical nursing. Studies concluded that high-quality humanized nursing could reduce the incidence of complications and improve patient satisfaction. Our department achieved great results and gain a proven experience in humanized care of patients with UAE in the perioperative period. Now the summary report is as follows. 


\section{Materials and Methods}

\subsection{General Information}

The subjects were patients who were treated with UAE in our gynecology department from November 2019 to November 2020. Inclusive criteria: (1) Not taking any anti-anxiety drugs; mentally normal with good expressive ability; (2) hospitalization time $>2$ days. Exclusive criteria: patients with serious chronic diseases such as diabetes, hypertension. Among the 90 selected patients aged 36 83 $(51.3 \pm 2.4)$ years, 36 cases were intramural fibroids, 24 cases were submucosal fibroids, and 26 cases were subserosal fibroids. Then the selected patients were divided into two groups according to a random drawing. In the control group, 45 patients were aged 36 79 (50.4 \pm 1.9$)$ years, 17 cases were intramural fibroids, 12 cases were submucosal fibroids, and 14 cases were subserosal fibroids. the diameter of fibroids was $2.1 \sim 6.1(4.0 \pm 1.2) \mathrm{cm}$. In the observation group, 45 patients were aged $38 \sim 83(53.2 \pm 3.3)$ years, 17 cases were intramural fibroids, 12 cases were submucosal fibroids, and 14 cases were subserosal fibroids; the diameter of fibroids was 2.2 5.9 $(3.8 \pm 1.1) \mathrm{cm}$. The general data of the two groups were comparable $(P>0.05)$. This study was approved by the ethics committee and all patients who participated in the study were informed and consented.

\subsection{Methods}

\subsubsection{Control Group}

Patients were treated with traditional care. The patient's condition is assessed by professional physicians, and the patient's basic physiological indicators were monitored by nurses. Besides, the patents were regularly organized to conduct simple pain knowledge education [5].

\subsubsection{Observation Group}

Based on traditional care, patients were taking more humanized care measures. (1) Create a suitable medical environment according to different patients, keep the ward quiet and well ventilated, and adjust an appropriate temperature at a different time of the day. (2) As most patients with uterine fibroids felt anxiety and depression because of the treatment effects on their physiological functions, nurses should be patient in psychological counseling. Explain in detail to patients the impact of negative emotions on the body, such as lowering immunity and a poorer prognosis, to ensure that patients could re-perceive negative emotions and consciously adjust their emotions [6]. Besides, assess the psychological status regularly, focused on the psychological care, and explains reasonable methods of emotional catharsis. (3) Posters related to the knowledge of uterine fibroids and profiles of the gynecology department team were hung in the corridors of the ward to eliminate the tension of patients. Also, brochures about knowledge of uterine fibroids and UAE with easy-to-understand pictures and text were printed for patients. (4) A workflow was set up to ensure professionalization in all aspects of nursing. The patients were educated to improve the compliance of taking medication. The drug delivery procedures were developed, and the infusion rate was adjusted according to the nature of the drugs used. The adverse reactions of the patients after taking the drugs were closely observed and the recovery status of the patients was also fed back to the professional physicians to adjust the drugs or dosage according to the patients' condition in time to ensure the therapeutic effect. (5) The patient was instructed to avoid hip flexion and knee flexion for 6 hours and to get out of bed as soon as possible after 24 hours. The patient was instructed on the importance of maintaining posture to alleviate discomfort, especially when discomfort occurred. (6) The patient might experience a feeling of cramping and bulging in the lower abdomen and lumbosacral area. This is because the uterine artery is blocked and the blood supply to the fibroids is stopped, which is caused by necrosis of the fibroids. Before the UAE treatment, the patient would be informed that pain might occur due to vascular embolism so that the patient could adapt to it in advance. Because each person has a different sensitivity, and their pain levels are also different. Nursing staff should observe the location and nature of the patient's pain in time, record the time, and help the physician handle it. The patient was also instructed in postoperative pain management skills, including how to breathe, assess the pain, and distract [7]. (7) Some patients may experience a small amount of vaginal bleeding or discharging dark red lumps, which should be told that this is normal during the post-operative period and that no special treatment is required. If the fibroids were found to be discharged out of the vagina, perineal care should be performed 2-3 times a day. If the fibroids were not completely discharged, the necrotic tissue out of the vagina can be removed after adequate disinfection of the vulva. Nurses should also instruct patients on how to perform perineal care.

\subsection{Observation Index}

After different modes of care intervention, anxiety, depression, satisfaction with care, quality of life, and adverse effects such as menorrhea, anemia, infection, and arterial thrombosis were compared between the two groups. (1) The quality of life was evaluated by the Quality of Life Scale (QOLS). The scale includes 6 major areas: religious beliefs, living environment, social relations, independent ability, mental status, and physical function. Each area is scored separately using a 5-level scoring method, which is $<60$ points, $60-69$ points respectively, $70-79$ points, $80-89$ points, $90-100$ points. The higher score means the better the quality of life. (2) The anxiety and depression degree was evaluated before being discharged from the hospital. Anxiety was evaluated by the Hamilton Anxiety Scale (HAMA), and depression was evaluated by the Hamilton Depression Scale (HAMD), the higher the score, the more serious it is. (3) Patients who were to be discharged from the hospital were given a questionnaire to conduct a nursing satisfaction survey, which was based on the evaluation of pain management, nursing quality, physicians' treatment, and other aspects, with a total score of 100. A higher score means better satisfaction. (4) The cases of menorrhea, anemia, infection, arterial thrombosis, and other 
adverse conditions were investigated.

\subsection{Statistical Methods}

SPSS 21.0 software was used for statistical analysis. Measurement data were expressed as mean \pm standard deviation $(\bar{x} \pm s)$, and comparison between groups was performed by $t$-test. Statistical data were expressed in terms of frequency and percentage (\%) when the chi-square test was used. The difference was statistically significant with $P<$ 0.05 .

\section{Results}

\subsection{Comparison of QOLS Between Two Groups}

The scores of religious beliefs, living environment, social relations, independent ability, mental status, and physical function of the observation group after nursing were significantly higher than those of the control group, and the differences were statistically significant $(P<0.05)$.

Table 1. Comparison of QOLS between two groups $(\bar{x} \pm s)$.

\begin{tabular}{|c|c|c|c|c|c|c|c|}
\hline Group & $n$ & $\begin{array}{l}\text { Religious } \\
\text { Beliefs } \\
\end{array}$ & $\begin{array}{l}\text { Living } \\
\text { Environment }\end{array}$ & Social Relations & $\begin{array}{l}\text { Independent } \\
\text { Ability }\end{array}$ & Mental Status & $\begin{array}{l}\text { Physical } \\
\text { Function }\end{array}$ \\
\hline Control Group & 45 & $34.51 \pm 5.57$ & $45.98 \pm 12.40$ & $55.02 \pm 12.82$ & $47.78 \pm 10.95$ & $46.91 \pm 13.21$ & $41.42 \pm 10.50$ \\
\hline Observation Group & 45 & $38.41 \pm 11.26$ & $51.80 \pm 11.28$ & $60.22 \pm 12.82$ & $57.02 \pm 13.25$ & $55.84 \pm 13.85$ & $51.62 \pm 12.87$ \\
\hline$t$ & & 2.088 & 2.330 & 1.882 & 3.607 & 3.132 & 4.118 \\
\hline$P$ & & $<0.05$ & $<0.05$ & $<0.05$ & $<0.05$ & $<0.05$ & $<0.05$ \\
\hline
\end{tabular}

\subsection{Comparison of HAMD Score and Satisfaction}

The score of HAMA and HAMD of the observation group was significantly higher than that of the control group, and the differences were statistically significant $(P<0.05)$. The

satisfaction of the observation group was significantly higher than that of the control group, and the differences were statistically significant $(P<0.05)$.

Table 2. Comparison of HAMA score, HAMD score, and satisfaction $(\bar{x} \pm s)$.

\begin{tabular}{lllll}
\hline Group & $\boldsymbol{n}$ & HAMA Score & HAMD Score & Satisfaction \\
\hline Control Group & 45 & $11.16 \pm 4.17$ & $11.91 \pm 4.33$ \\
Observation Group & 45 & $7.56 \pm 2.83$ & $8.78 \pm 3.05$ & $84.60 \pm 6.80$ \\
$t$ & & 4.787 & 3.967 & $91.80 \pm 4.95$ \\
$P$ & & $<0.05$ & $<0.05$ & 5.742 \\
\hline
\end{tabular}

\subsection{Comparison of Rate of Adverse Complications}

The rate of adverse complications of the observation group was significantly lower than that of the control group, and the differences were statistically significant $(P<0.05)$.

Table 3. Comparison of rate of adverse complications (cases/\%).

\begin{tabular}{|c|c|c|c|c|c|c|}
\hline Group & $n$ & Amenorrhea & Anemia & Infection & Arterial Thrombosis & Total \\
\hline Control Group & 45 & $3(6.6)$ & $4(8.8)$ & $3(6.6)$ & $2(4.4)$ & $12(26.7)$ \\
\hline Observation Group & 45 & 0 & $1(2.2)$ & $1(2.2)$ & 0 & $2(4.4)$ \\
\hline$\chi^{2}$ & & & & & & 8.459 \\
\hline$P$ & & & & & & $<0.05$ \\
\hline
\end{tabular}

\section{Discussion}

With the advancement of with advances in interventional radiology techniques, UAE has been widely used to treat uterine fibroids by injecting permanent embolic pellets to block the blood flow to the uterine fibroids' nutritive arteries, causing them to become ischemic and shrink [8]. Although UAE has the advantages of being less invasive and preserving the patient's uterus [9], factors such as pain, vaginal bleeding, and psychological stress caused by embolization can cause some stimulation to the body, and most patients have negative emotions in the perioperative period [10]. In this study, to achieve a humanized care model, a detailed nursing plan was developed based on the general information of the patients and the results of the analysis of their conditions. Patient communication with the patients and their families could ensure that the nursing plan was effectively implemented [11]. Most patients were initially unacceptable to their condition and feared that the UAE treatment would have an impact on their reproductive function, resulting in anxious psychological emotions and mood swings that adversely affect recovery [12, 13]. In response to this, psychological care should be provided to patients to understand the psychological changes. The psychological problems should be promptly guided and mediated to improve negative emotions so that patients have a concrete understanding of uterine fibroids and increase their confidence in overcoming the disease. In terms of complications care, nursing staff are required to prevent complications such as pain, nausea and vomiting, infection, irregular vaginal bleeding, and arterial thrombosis, and implement symptomatic treatment in time to prevent the 
deterioration of the condition due to complications and delay the recovery time of the patient $[14,15]$. Compared with traditional nursing care, humanized care has the characteristics of "comprehensiveness, systematization and scientificity", which can ensure that patients' physiological and psychological needs can be met and provide patients with better nursing services.

\section{Conclusion}

In conclusion, Humanized nursing is a nursing model that shifts from a biological model to a biological-social-psychological model. Humanized nursing not only focuses on solving patients' disease pain and physical needs but also pays attention to other aspects of patients' psychology. It not only satisfies the needs of treating disease but also satisfies the needs of human dignity. The nurse needs to conduct a comprehensive nursing assessment of the patient and based on the evaluation and diagnosis results, formulate nursing plans from all aspects that are conducive to the rehabilitation of patients, including psychological counseling in psychological care and other aspects. These inevitably require nurses to have a wide range of knowledge, including psychology, ethics, humanities, and other diverse knowledge, to better master the art of communication. The application of a humanized nursing model for the care of patients with UAE for uterine fibroids can effectively alleviate and eliminate negative perioperative emotions and significantly improve depression, which in turn is conducive to improving their postoperative quality of life, with high patient satisfaction and fewer cases of adverse reactions, with a high degree of safety, and is worth promoting in the clinic.

\section{References}

[1] ZHIYING X, YICHUN G, HONGFANG F, et al. Effect of intramural fibroid and uterine artery blood flow parameters on the pregnancy and perinatal outcome of IVF/ICSI patients [J]. Chinese Journal of Clinical Obstetrics and Gynecology, 2017, 18 (6): 498-502.

[2] CHAPIRO J, DURAN R, LIN M, et al. Three-Dimensional Quantitative Assessment of Uterine Fibroid Response after Uterine Artery Embolization Using Contrast-Enhanced MR Imaging [J]. J Vasc Interv Radiol, 2015, 26 (5): 670-678.

[3] KIM J H. Analysis of the in vitro effects of di- (2-ethylhexyl) phthalate exposure on human uterine leiomyoma cells [J]. Exp Ther Med, 2018, 15 (6): 4972-4978.

[4] RAN L J, HUI W L. Trends and related issues in the treatment of patients with uterine fibroids with preserved reproductive function [J]. Chinese Journal of Clinical Obstetrics and Gynecology, 2014, 15 (6): 555-556.
[5] HUA J L, LING H Y, HUA Y G, et al. Value of cervical cold knife conization combined with ligation of the lower branch of the uterine artery in the treatment of high-grade cervical intraepithelial neoplasia $[\mathrm{J}]$. Chinese Journal of Clinical Obstetrics and Gynecology, 2017, 18 (5): 443-444.

[6] KOL'TSOVA A S, PENDinA A A, EFIMOVA O A, et al. Differential DNA Hydroxymethylation in Human Uterine Leiomyoma Cells Depending on the Phase of Menstrual Cycle and Presence of MED12 Gene Mutations [J]. Bull Exp Biol Med, 2017, 163 (5): 646-649.

[7] JUAN L R, HUA L Q, BO Y C, et al. Postoperative quality of life and factors influencing it in patients with multiple uterine fibroids treated by combined laparoscopic uterine artery embolization [J]. Chinese Journal of Clinical Research, 2015, 28 (11): 1501-1503.

[8] RESNICK N J, KIM E, PATEL R S, et al. Uterine artery embolization using a transradial approach: initial experience and technique [J]. J Vasc Interv Radiol, 2014, 25 (3): 443-447.

[9] KONSTANTATOS A H, KAVNOUDIAS H, STEGEMAN J R et al. A randomized, double-blind, placebo-controlled study of preemptive oral oxycodone with morphine patient-controlled anesthesia for postoperative pain management in patients undergoing uterine artery embolization for symptomatic uterine fibroids [J]. Cardiovasc Intervent Radiol, 2014, 37 (5): 1191-1197.

[10] XIA L H. Influences of nursing intervention on stress and quality of sex life for patients with hysteromyoma resection surgery [J]. Chinese Journal of Clinical Oncology and Rehabilitation, 2016, 23 (6): 757-759.

[11] WEI L I, CAIFANG N I, JIANWEI Z, et al. Prophylactic bilateral uterine artery catheterization and embolization in treatment of pernicious placenta previa and accrete $[\mathrm{J}]$. Chinese Journal of Interventional Imaging and Therapy, 2017, 14 (6): 339-342.

[12] SCHEURIG-MUENKLER C, POWERSKI M J, MUELLER J $\mathrm{C}$, et al. Radiation exposure during uterine artery embolization effective measures to minimize dose to the patient [J]. Cardiovasc Intervent Radiol, 2015, 38 (3): 613-622.

[13] WENLONG Z, CHENGWEI L, FANGWEI Y, et al. Far and Near Effect of Self-Made Huoxue Huayu Formula Combined with Uterine Arterial Embolization and Methotrexate in Treatment of Placenta Implantation [J]. Chinese Archives of Traditional Chinese Medicine, 2018, 36 (4): 1019-1021.

[14] LIU Y, LU D, SHENG J, et al. Identification of TRADD as a potential biomarker in human uterine leiomyoma through iTRAQ based proteomic profiling [J]. Mol Cell Probes, 2017, 36: $15-20$.

[15] LIANGLIANG B, TIANTIAN L I, ZONGMING L I, et al. Necessity of prophylactic uterine artery embolization before curettage in treatment of cesarean scar pregnancy [J]. Chinese Journal of Interventional Imaging and Therapy, 2018, 15 (1): 47-50. 